\title{
Ângulo de Böhler-comparação entre o pré e pós-operatório nas fraturas intra-articulares desviadas do calcanhar*
}

\section{Böhler's Angle-Comparison Between the Pre- and Postoperative in Displaced Intra-Articular Calcaneal Fractures}

\author{
Pedro José Labronici ${ }^{1,2}$ Guilherme Guerra Pinheiro de Faria ${ }^{1}$ Bruno Miranda Pedro ${ }^{1}$ \\ Marcos Donato Franco de Araújo Serra ${ }^{1}$ Robinson Esteves Santos Pires ${ }^{3}$ Jorge Luiz Tamontini ${ }^{4}$ \\ 1 Serviço de Ortopedia e Traumatologia, Hospital Santa Teresa, \\ Petrópolis, RJ, Brasil \\ 2 Universidade Federal Fluminense, Niterói, RJ, Brasil \\ 3 Universidade Federal de Minas Gerais, Belo Horizonte, MG, Brasil \\ ${ }^{4}$ Instituto de Ortopedia e Traumatologia, Hospital Dr. Bartolomeu \\ Tacchini, Bento Gonçalves, RS, Brasil
Address for correspondence Pedro José Labronici, Serviço de Ortopedia e Traumatologia, Hospital Santa Teresa, Petrópolis, RJ, Brasil (e-mail: plabronici@globo.com). \\ Rev Bras Ortop 2019;54:156-164.
}

\section{Resumo \\ Palavras-chave \\ - calcâneo \\ - fraturas ósseas \\ - fraturas intra- articulares \\ - radiografias}

Objetivo Comparar a variação dos resultados das medidas radiográficas do ângulo de Böhler, no pré e pós-operatório, em fraturas com dois tipos de desvio: graves e moderadas.

Métodos: O ângulo de Böhler foi analisado retrospectivamente em 31 radiografias pré e pós-operatórias de fraturas do calcâneo. Quatro pacientes eram do sexo feminino (6,5\%) e 26 do masculino (83,9\%), entre 23 e 72 anos, média de 44,5 .

Resultados As medidas pré e pós-operatória demonstraram que o ângulo de Böhler após a cirurgia foi significativamente maior do que o ângulo de Böhler pré-operatório $(\mathrm{p}$-valor $=0,000)$. Nas análises intraobservador e global, o ângulo de Böhler pósoperatório foi, em média, 10,6 graus maior do que no pré-operatório. O ângulo pósoperatório foi, em média, $108 \%$ maior do que o ângulo pré-operatório. No global, a concordância entre os avaliadores é excelente, tanto em relação ao valor pontual estimado $(0,98)$ quanto em relação ao intervalo de confiança do ICC.

Conclusão $\mathrm{Na}$ análise global, verificou-se que as medidas do ângulo de Böhler no pósoperatório são, em média, significativamente maiores do que as do ângulo préoperatório. Quanto mais distante da faixa de normalidade (20 a 40 graus) estiver o ângulo pré-operatório, maior a diferença no ângulo após a cirurgia. Quando o ângulo pré-operatório está na faixa de normalidade, o ângulo pós-operatório será, em média, 1,28 vez o ângulo pré-operatório; se o ângulo de Böhler pré-operatório estiver fora da

\footnotetext{
Trabalho desenvolvido no Serviço de Ortopedia e Traumatologia, Hospital Santa Teresa, Petrópolis, RJ, Brasil. Publicado originalmente por Elsevier Ltda.
}

received

August 16, 2017

accepted

December 7, 2017
DOI https://doi.org/

10.1016/j.rbo.2017.12.005. ISSN 0102-3616.
Copyright $(2019$ by Sociedade Brasileira License terms de Ortopedia e Traumatologia. Published by Thieme Revnter Publicações Ltda, Rio de Janeiro, Brazil 


\begin{abstract}

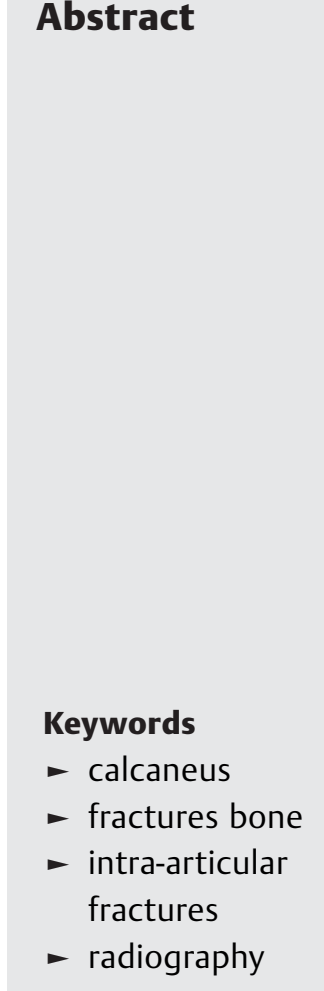

Objective To compare pre- and postoperative variation of radiographic measurements of the Böhler angle (BA) in fractures with two types of deviations: severe and moderate.

Methods Pre- and postoperative BAs in 31 calcaneal fracture radiographs were retrospectively analyzed. A total of 4 patients were female (6.5\%) and 26 were male (83.9\%), with age ranging from 23 to 72 years old, and a mean age of 44.5 years old. Results The results show that the postoperative BA was significantly larger than the preoperative $\mathrm{BA}(p=0.000)$. At the intraevaluator and overall assessments, the postoperative $B A$ was, on average, $10.6^{\circ}$ higher than the preoperative measure. The postoperative angle was, on average, $108 \%$ higher than the preoperative angle. In the global assessment, the agreement between evaluators was excellent, both regarding the estimated point value (0.98) and the intraclass correlation (ICC) confidence interval (CI).

Conclusion In the global analysis, the postoperative BAs were, on average, significantly higher than the preoperative measurements. The farther from the normal range $\left(20^{\circ}\right.$ to $\left.40^{\circ}\right)$ the preoperative angle is, the greater the difference after the surgery. When the preoperative angle was normal, the postoperative angle was, on average, 1.28 times the preoperative measurement. If the preoperative BA was abnormal, the postoperative angle was, on average, 17.3 times the preoperative measurement. It was demonstrated that more severe fractures present better anatomic results when compared with moderate fractures. The present study also confirms a good interobserver correlation for the BA.
\end{abstract}

faixa de normalidade, o ângulo pós-operatório será, em média, 17,3 vezes o ângulo préoperatório. Ficou demonstrado que as fraturas mais graves apresentam melhores resultados anatômicos quando comparadas com as fraturas moderadas. $\mathrm{O}$ estudo também confirmou uma boa correlação interobservador para o ângulo de Böhler.

\section{Introdução}

O calcâneo é o osso mais frequentemente traumatizado e representa $60 \%$ das fraturas do retropé. Estas fraturas compreendem cerca de $1 \%$ a $2 \%$ de todas as fraturas e aproximadamente $75 \%$ apresentam um componente intra-articular, com importantes consequências para os pacientes. ${ }^{1}$

$O$ ângulo de Böhler $(\mathrm{AB})$ é o ângulo complementar formado por duas linhas: (a) uma linha entre a região mais alta do processo anterior e a parte mais alta da superfície articular posterior e (b) uma linha entre o mesmo ponto sobre a superfície articular posterior e o ponto mais superior da tuberosidade do calcâneo. Normalmente, o AB varia entre 20。 a $40{ }^{2-6}{ }^{2-6}$ frequentemente usado, em radiografias de perfil, para avaliar o grau e a gravidade da deformidade da fratura intra-articular desviada do calcâneo e auxilia a confirmar o resultado da redução em radiografias do pós-operatório. ${ }^{7-11}$ Alguns autores sugerem que a restauração do $A B$ melhora os resultados e indica o valor prognóstico no pós-operatório de fratura e na artrodese da articulação subtalar. ${ }^{2-6,12,13}$

Segundo a literatura, o AB auxilia no resultado clínico ao correlacionar as variações entre as medidas do pré e pósoperatório. ${ }^{8-11}$ Knight et al ${ }^{14}$ demonstraram que artigos sobre o AB têm mostrado boa confiabilidade intraobservador. 0 objetivo deste trabalho foi comparar a variação dos resultados das medidas radiográficas do $\mathrm{AB}$, no pré e pós-operatório, em fraturas com dois tipos de desvio: graves e moderadas.

\section{Material e métodos}

De abril de 2015 a junho de 2017, foram analisadas, retrospectivamente, 31 radiografias pré e pós-operatórias de fraturas do calcâneo. $\mathrm{O}$ estudo foi analisado e autorizado pelo Comitê de Ética do Hospital e não houve a necessidade do Termo de Consentimento Livre e Esclarecido (TCLE), pois os dados foram extraídos dos prontuários e de radiografias dos pacientes.

Os fatores de inclusão foram pacientes com fraturas desviadas do calcâneo, maiores de 18 anos e tratados com menos de três semanas de fratura. Os fatores de exclusão foram casos em que as radiografias estavam incompletas ou de baixa qualidade para fazer as medições, fraturas prévias ou patológicas, fraturas expostas, contraindicação médica para cirurgia e fraturas do calcâneo com fraturas concomitantes dos ossos do tornozelo ou pé. Todos os pacientes foram operados pelo acesso lateral estendido.

$\mathrm{O} A \mathrm{~B}$ foi medido nas radiografias do pré e pós-operatório dos pacientes com fraturas do calcâneo. $\mathrm{O}$ ângulo foi considerado dentro de um limite aceitável quando se encontrava entre $20 \circ$ a 40 o e foram analisados por dois pesquisadores independentes (residentes do $3 \circ$ ano). 
Tabela 1 Distribuição de frequências da idade dos pacientes

\begin{tabular}{|c|c|c|c|c|c|c|}
\hline \multirow{2}{*}{$\begin{array}{l}\text { Idade } \\
\text { (anos) }\end{array}$} & \multicolumn{2}{|l|}{ Global } & \multicolumn{2}{|l|}{ Feminino } & \multicolumn{2}{|l|}{ Masculino } \\
\hline & $\begin{array}{l}\text { Frequência } \\
\text { absoluta }\end{array}$ & $\begin{array}{l}\text { Frequência } \\
\text { relativa }\end{array}$ & $\begin{array}{l}\text { Frequência } \\
\text { absoluta }\end{array}$ & $\begin{array}{l}\text { Frequência } \\
\text { relativa }\end{array}$ & $\begin{array}{l}\text { Frequência } \\
\text { absoluta }\end{array}$ & $\begin{array}{l}\text { Frequência } \\
\text { relativa }\end{array}$ \\
\hline 22 a 30 & 3 & $10,0 \%$ & 1 & $25,0 \%$ & 2 & $7,7 \%$ \\
\hline 31 a 39 & 6 & $20,0 \%$ & 0 & $0,0 \%$ & 6 & $23,1 \%$ \\
\hline 40 a 48 & 13 & $43,3 \%$ & 0 & $0,0 \%$ & 13 & $50,0 \%$ \\
\hline 49 a 57 & 6 & $20,0 \%$ & 2 & $50,0 \%$ & 4 & $15,4 \%$ \\
\hline 58 a 64 & 0 & $0,0 \%$ & 0 & $0,0 \%$ & 0 & $0,0 \%$ \\
\hline 65 a 73 & 2 & $6,7 \%$ & 1 & $25,0 \%$ & 1 & $3,8 \%$ \\
\hline
\end{tabular}

Tabela 2 Principais estatísticas da distribuição de idade dos pacientes

\begin{tabular}{|l|l|l|l|l|l|}
\hline Média & Mediana & DP & Mínimo & Máximo & CV \\
\hline 44,5 & 46,0 & 11,3 & 23,0 & 72,0 & 0,25 \\
\hline
\end{tabular}

Abreviações: CV, coeficiente de variação; DP, desvio-padrão

A amostra-base desta pesquisa foi formada por 31 pacientes, quatro do gênero feminino $(6,5 \%)$ e 26 do masculino $(83,9 \%)$. Dos 31 pacientes, de um não havia registro das informações sobre gênero e idade. A distribuição de frequências da idade dos pacientes por gênero e a global podem ser vistas na $\boldsymbol{-}$ Tabela 1 e a distribuição de idade na $\boldsymbol{- T a b e l a ~} \mathbf{2}$. Os pacientes apresentaram entre 23 e 72 anos e resultaram em uma média de 44,5 anos, mediana de 46 , e desvio-padrão de 11,3 , com coeficiente de variação igual a 0,25 , evidenciou-se moderada variabilidade entre as idades dos 31 pacientes. A faixa etária foi de 40 a 48 anos, na qual se concentravam $43,3 \%$ da amostra.

\section{Metodologia}

As variáveis deste estudo são as medidas do ângulo de Böhler, feitas por dois avaliadores, a partir de exames radiográficos de fraturas desviadas do calcâneo graves e moderadas. A partir dos dados coletados, foi construído um banco de dados analisado pelo programa SPSS (Statistical Package for the Social Science), versão 22.0, e pelo aplicativo Microsoft Excel 2007.

Para caracterização da amostra e análise descritiva do comportamento das variáveis, os dados foram sintetizados por meio do cálculo de estatísticas descritivas (média, mediana, mínimo, máximo, desvio-padrão, coeficiente de variação - CV), gráficos descritivos e distribuições de frequência. A variabilidade da distribuição de uma variável foi considerada baixa se $C V<0,20$; moderada se $0,20 \leq C V$ $<0,40$ e alta se $C V \geq 0,40$.

Na análise inferencial, a hipótese de normalidade da distribuição de uma medida foi verificada pelo teste de Kolmogorov-Smirnov e pelo teste de Shapiro-Wilk. A distribuição em teste foi considerada normal quando ambos os testes assim o concluíram. As medidas do AB pré-operatórios foram comparadas com as medidas dos ângulos pós-opera- tórios e as medidas dos ângulos foram comparadas entre os dois avaliadores. Quando as duas medidas a serem comparadas tinham distribuições normais, elas foram comparadas em pares pelo teste $t$ de Student pareado. Quando pelo menos uma das medidas a serem comparadas não tinha distribuição normal, duas medidas pareadas foram comparadas pelo teste de Wilcoxon.

Foi feita a análise de concordância entre as medidas dos dois avaliadores através da quantificação da concordância bruta (percentual de casos em que as duas medidas são iguais, ou seja, com diferença entre as duas medidas D, igual a 0 ) e por meio do cálculo do coeficiente de correlação intraclasse (ICC). O ICC expressa a proporção de variabilidade total, que é devida à variabilidade entre as unidades. No caso de se avaliar a concordância entre duas medidas, como a concordância entre o ângulo medido pelo avaliador $1 \mathrm{e} o$ avaliador 2, o ICC pode ser interpretado como uma medida de concordância, que mede o grau de afastamento das duas medidas à reta de 45 graus, no qual teria concordância perfeita, pois ambas as medidas seriam iguais. O ICC foi calculado no modelo Anova de efeitos mistos (Two Way Mixed) e o interesse de estudo foi o de "análise de consistência". A classificação da concordância a partir do ICC foi baseada na classificação:

$$
\begin{aligned}
& 0,00 \leq \text { ICC } \leq 0,20=\text { concordância pobre, } \\
& 0,20<\text { ICC } \leq 0,40=\text { concordância razoável } \\
& 0,40<\text { ICC } \leq 0,60=\text { concordância boa } \\
& 0,60<\text { ICC } \leq 0,80=\text { concordância muito boa } \\
& 0,80<\text { ICC } \leq 1,00=\text { concordância excelente }
\end{aligned}
$$

A estimação da imprecisão dos estimadores de ICC foi analisada pelo intervalo de confiança do ICC no nível de $95 \%$ e a significância foi avaliada pelo teste F para o ICC. A concordância foi considerada significativamente boa se o ICC fosse significativamente não nulo e se o seu valor pontual e todos os valores do intervalo de confiança ao nível de $95 \%$ de confiança fossem, no mínimo, do nível de "boa concordância".

Todas as discussões consideraram nível de significância máximo de $5 \%(0,05)$, ou seja, foi adotada a seguinte regra de decisão nos testes: rejeição da hipótese nula sempre que o pvalor associado ao teste fosse menor do que 0,05 . Nos testes que forneceram p-valor assintótico e exato, foi considerado o p-valor exato. 
Tabela 3 Testes de normalidade para as distribuições do ângulo de Böhler, pré e pós-operatório, para cada avaliador e global

\begin{tabular}{|l|l|l|l|l|}
\hline Avaliador & Ângulo & $\begin{array}{l}\text { p-valor do } \\
\text { teste de } \\
\text { Kolmogorov- } \\
\text { Smirnov }\end{array}$ & $\begin{array}{l}\text { p-valor do } \\
\text { teste de } \\
\text { Shapiro- } \\
\text { Wilk }\end{array}$ & $\begin{array}{l}\text { Distribuição } \\
\text { normal }\end{array}$ \\
\hline 1 & Pré & 0,200 & 0,196 & Sim \\
\hline & Pós & 0,200 & 0,987 & Sim \\
\hline 2 & Pré & 0,200 & 0,61 & Sim \\
\hline & Pós & 0,200 & 0,922 & Sim \\
\hline Global & Pré & 0,200 & 0,051 & Sim \\
\hline & Pós & 0,200 & 0,936 & Sim \\
\hline
\end{tabular}

\section{Resultados}

\section{Análise descritiva das medidas dos ângulos}

A - Tabela 3 demonstra os p-valores dos testes de normalidade para as distribuições do ângulo de Böhler medidos pelos dois avaliadores e para a distribuição global (ou seja, independentemente do avaliador). A partir dos p-valores apresentados, todos maiores do que $5,0 \%$, conclui-se que todas as medidas do $\mathrm{AB}$, pré e pós-operatórias, para ambos os avaliadores e no global, seguem distribuição normal. Portanto, qualquer análise inferencial que comparasse o $\mathrm{AB}$ foi feita por abordagem paramétrica.

As principais estatísticas das distribuições das medidas do $\mathrm{AB}$ nos dois momentos, para cada avaliador e global (independentemente do avaliador), são exibidas na - Tabela 4. A partir dos coeficientes de variação $(\mathrm{CV})$, todos maiores do que 0,20 , observa-se que as medidas do $A B$ apresentam alta variabilidade na amostra. As distribuições do AB para cada avaliador e global são ilustradas nos gráficos boxplot, exibidos na - Fig. 1. No boxplot das medidas do ângulo pósoperatório do avaliador 2, é possível identificar que o valor máximo de 50 graus é um outlier, ou seja, um valor discrepante (o) dos demais pacientes. Os gráficos e as estatísticas mostram que o ângulo aumentou após a cirurgia e o efeito é significativo. $O$ teste $t$ de Student pareado para as medidas pré e pós-operatória demonstrou que o $A B$ após a cirurgia é, de fato, significativamente maior do que o $A B$ pré-operatório ( $\mathrm{p}$-valor $=0,000 \mathrm{em}$ todas as comparações).

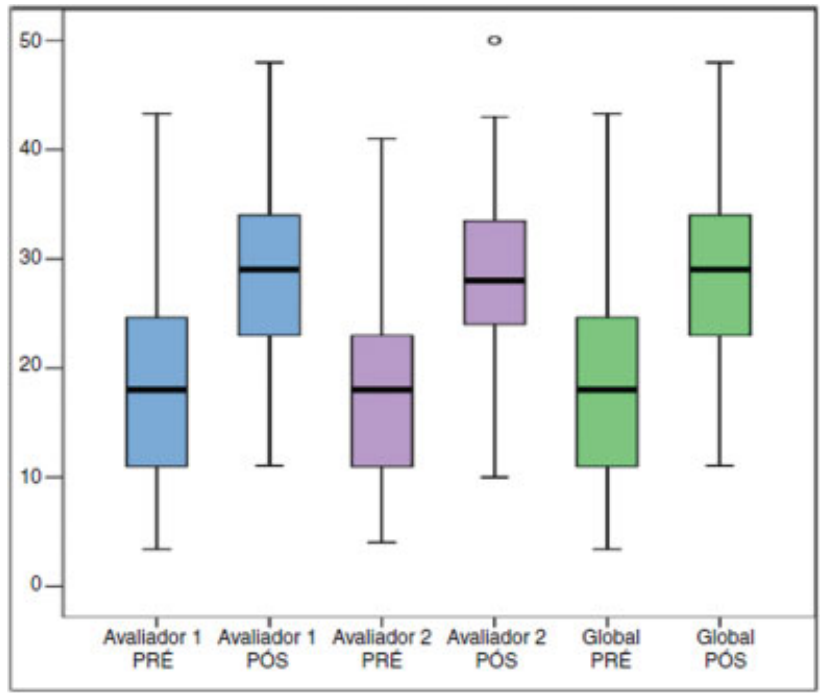

Fig. 1 Distribuições das medidas do ângulo de Böhler pré e pós para cada avaliador e global.

Para os avaliadores e no global, o AB pós-operatório é, em média, 10,6 graus maior do que no pré-operatório. As estatísticas da diferença entre os dois ângulos, por avaliador e global, podem ser vistas na - Tabela 5. Para duas observações do avaliador 1, o ângulo pós-operatório foi menor do que o ângulo pré-operatório. A variabilidade da diferença entre os dois ângulos é muito alta (CV maiores do que 0,80 ) e as maiores diferenças encontradas, maiores do que 40 graus, são muito atípicas e constituem outliers nas distribuições (-Fig. 2). As diferenças entre as medidas dos ângulos não seguem distribuição normal, pois apresentaram p-valores menores do que $5 \%$, para ambos os testes de normalidade e para ambos os avaliadores. Na comparação das diferenças entre o $\mathrm{AB}$ pré e pós-operatórios dos dois avaliadores pelo teste de Wilcoxon, obteve-se $\mathrm{p}$-valor $=0,761$. Conclui-se, portanto, que não havia diferença significativa entre as variações dos ângulos entre os dois avaliadores.

A - Tabela 6 mostra as estatísticas da diferença entre os dois ângulos, relativa (percentual) ao ângulo pré-operatório, por avaliador e global. Para os avaliadores e no global, o ângulo pós-operatório é, em média, $108,1 \%$ maior do que o ângulo pré-operatório. A variabilidade da diferença relativa entre os dois ângulos é muito alta (CV maiores do que 1,5$)$ e

Tabela 4 Principais estatísticas das distribuições das medidas do ângulo de Böhler, pré e pós-operatório, para cada avaliador e global

\begin{tabular}{|l|l|l|l|l|l|l|l|l|}
\hline Avaliador & Avaliação & Média & Mediana & Mínimo & Máximo & DP & CV & P-valor \\
\hline 1 & Pré & 18,5 & 18,0 & 3,4 & 43,3 & 9,4 & 0,51 & 0,000 \\
\hline & Pós & 28,9 & 29,0 & 11,1 & 48,0 & 8,3 & 0,29 & \\
\hline 2 & Pré & 18,2 & 18,0 & 4,0 & 41,0 & 8,9 & 0,49 & 0,000 \\
\hline & Pós & 28,8 & 28,0 & 10,0 & 50,0 & 8,8 & 0,31 & \\
\hline Global & Pré & 18,4 & 18,0 & 3,4 & 43,3 & 9,1 & 0,49 & 0,000 \\
\hline & Pós & 29,0 & 29,0 & 10,0 & 50,0 & 8,5 & 0,29 & \\
\hline
\end{tabular}

${ }^{a}$ Teste $t$ de Student pareado comparou as medidas pré e pós-operatórias. Abreviações: CV, coeficiente de variação; DP, desvio-padrão 
Tabela 5 Principais estatísticas da diferença entre as medidas do ângulo de Böhler pré e pós-operatórias, para cada avaliador e global

\begin{tabular}{|l|l|l|l|l|l|l|}
\hline Avaliador & Média & Mediana & Mínimo & Máximo & DP & CV \\
\hline 1 & 10,6 & 9,8 & $-2,5$ & 42,5 & 9,2 & 0,87 \\
\hline 2 & 10,6 & 8,0 & 1,0 & 46,0 & 8,6 & 0,81 \\
\hline Global & 10,6 & 8,0 & $-2,5$ & 46,0 & 8,8 & 0,83 \\
\hline
\end{tabular}

Abreviações: CV, coeficiente de variação; DP, desvio-padrão

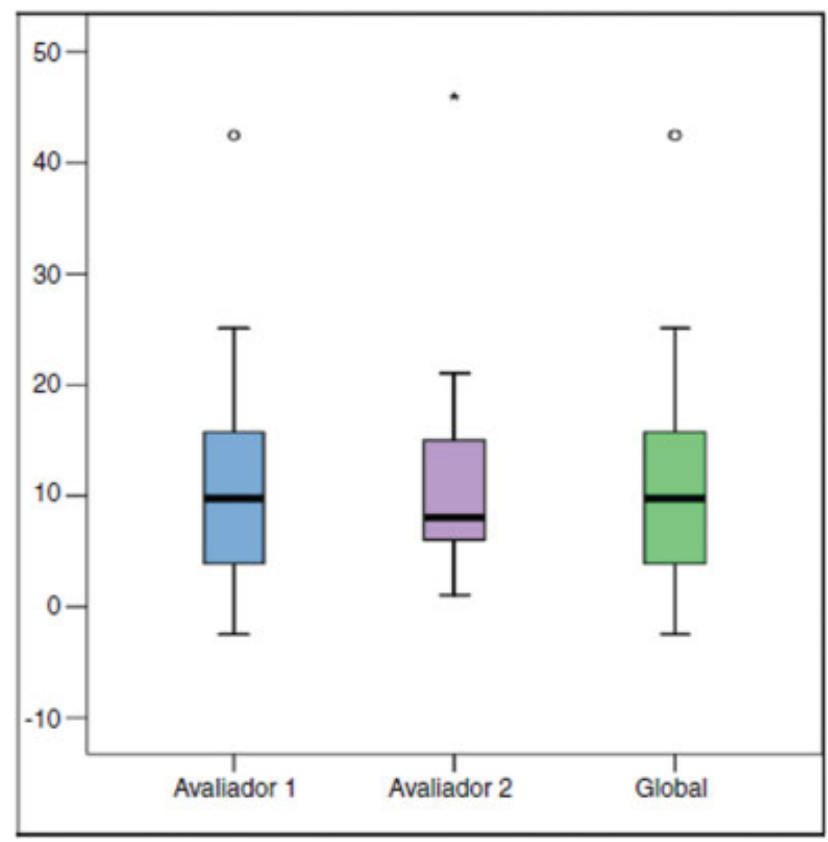

Fig. 2 Distribuições das diferenças entre as medidas do ângulo de Böhler pré e pós para cada avaliador e global.

as maiores diferenças encontradas, maiores do que 40 graus, constituem outliers nas distribuições das avaliações. Os boxplots das diferenças relativas entre os ângulos pré e pós-operatório são exibidos na - Fig. 3. A - Fig. 4 mostra que as diferenças acima de $250 \%$ do ângulo pré-operatório são valores atípicos, outliers na distribuição. As diferenças relativas entre as medidas dos ângulos não seguem distribuição normal, pois apresentaram p-valores menores do que $5 \%$, para ambos os testes de normalidade e para ambos os avaliadores. Na comparação das diferenças relativas entre os ângulos pré e pós-operatórios dos dois avaliadores pelo teste de Wilcoxon, obteve-se $\mathrm{p}$-valor $=0,666$ e conclui-se que não havia diferença significativa entre as variações relativas observadas nos ângulos pelos dois avaliadores.

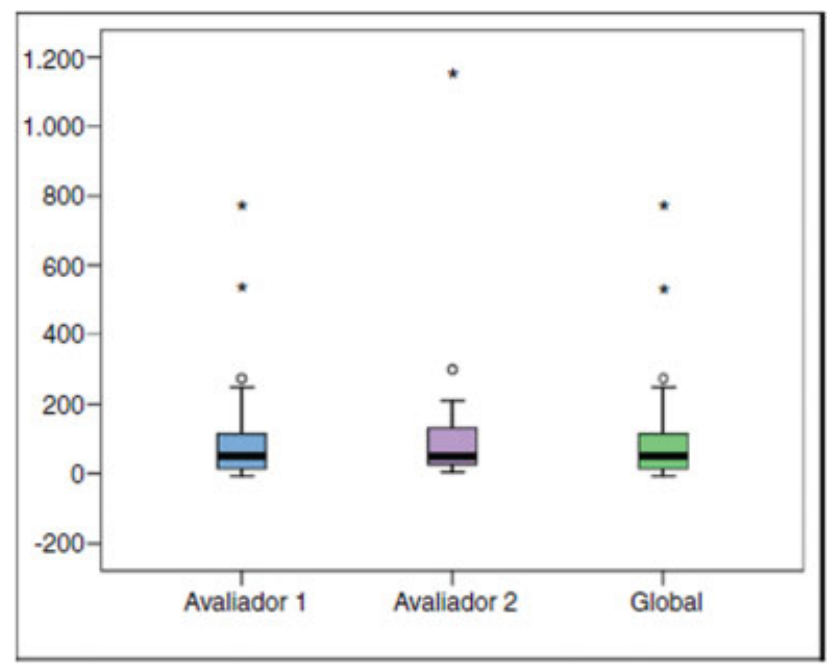

Fig. 3 Distribuições das diferenças relativas (\%) entre as medidas do ângulo de Böhler pré e pós para cada avaliador e global.

A - Fig. 4 mostra a relação entre a diferença relativa do ângulo e a medida do $A B$ pré-operatório para as medidas dos avaliadores 1 e 2 . O gráfico mostra uma curva de função inversa que explica bem o comportamento das duas variáveis $[(\mathrm{R})]^{2}>0,50$.

A - Fig. 5 mostra a relação entre a diferença relativa do ângulo e a medida do $A B$ pré-operatório para as medidas globais, sem discriminar os avaliadores. A relação global entre a diferença relativa do ângulo e a medida pré-operatória do $\mathrm{AB}$ também é bem explicada por uma função inversa $[(\mathrm{R})]^{2}=0,50$.

A partir dos gráficos das - Figs. 4 e 5 e dos dados, é possível verificar que a diferença relativa observada no ângulo após a cirurgia é maior para menores valores do ângulo pré-operatório e decresce à medida que o ângulo pré-operatório aumenta. Quanto mais distante da faixa de normalidade, de 20 a 40 graus, estiver o ângulo pré-operatório, maior a diferença relativa observada no ângulo após a cirurgia. Quando o ângulo préoperatório está na faixa de normalidade de 20 a 40 graus, a

Tabela 6 Principais estatísticas da diferença entre as medidas do ângulo de Böhler pré e pós-operatórias, relativa ao ângulo précirúrgico, para cada avaliador e global

\begin{tabular}{|l|l|l|l|l|l|l|}
\hline Avaliador & Média & Mediana & Mínimo & Máximo & DP & CV \\
\hline 1 & $108,1 \%$ & $50,6 \%$ & $-5,8 \%$ & $772,7 \%$ & $165,3 \%$ & 1,52 \\
\hline 2 & $108,9 \%$ & $50,0 \%$ & $4,9 \%$ & $1150,0 \%$ & $165,3 \%$ & 1,51 \\
\hline Global & $108,5 \%$ & $50,3 \%$ & $-5,8 \%$ & $1150,0 \%$ & $185,0 \%$ & 1,70 \\
\hline
\end{tabular}

Abreviações: CV, coeficiente de variação; DP, desvio-padrão 


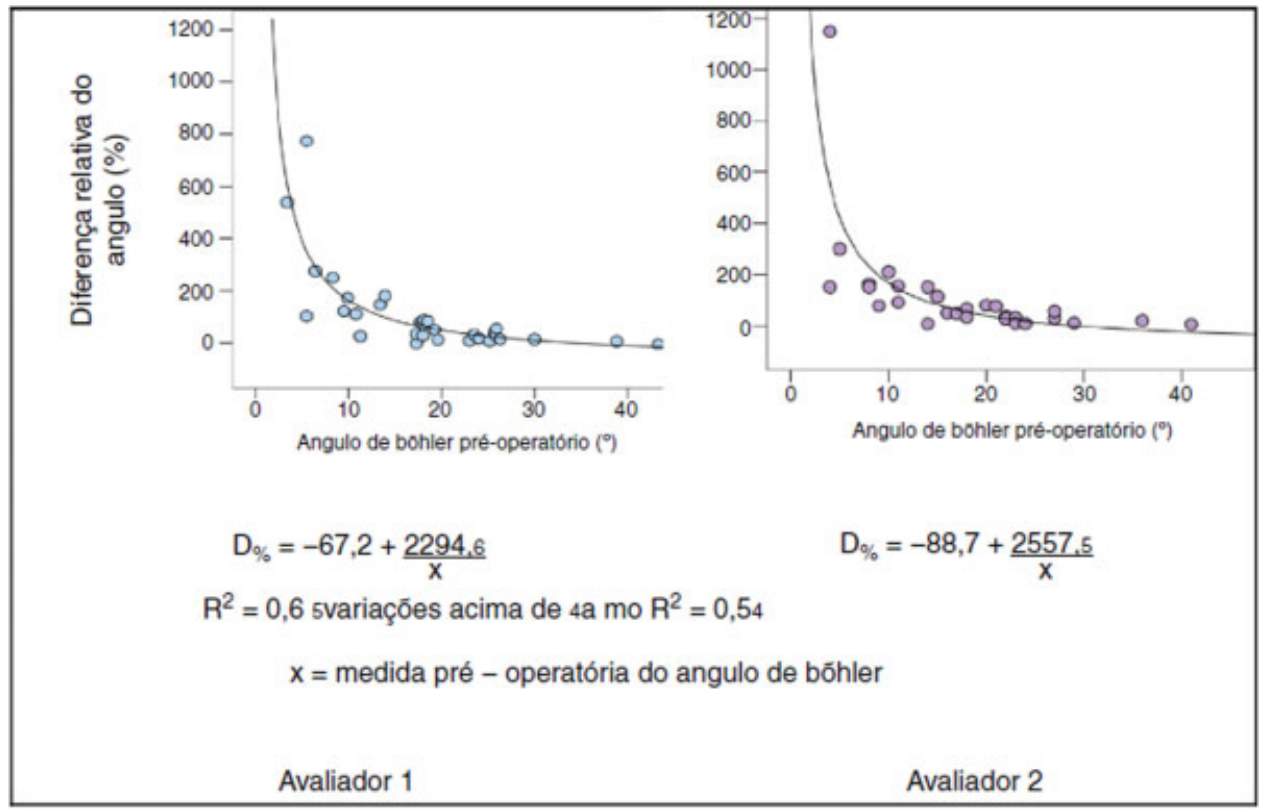

Fig. 4 Relação entre a diferença relativa do ângulo e a medida do ângulo de Böhler pré-operatório para os avaliadores 1 e 2.

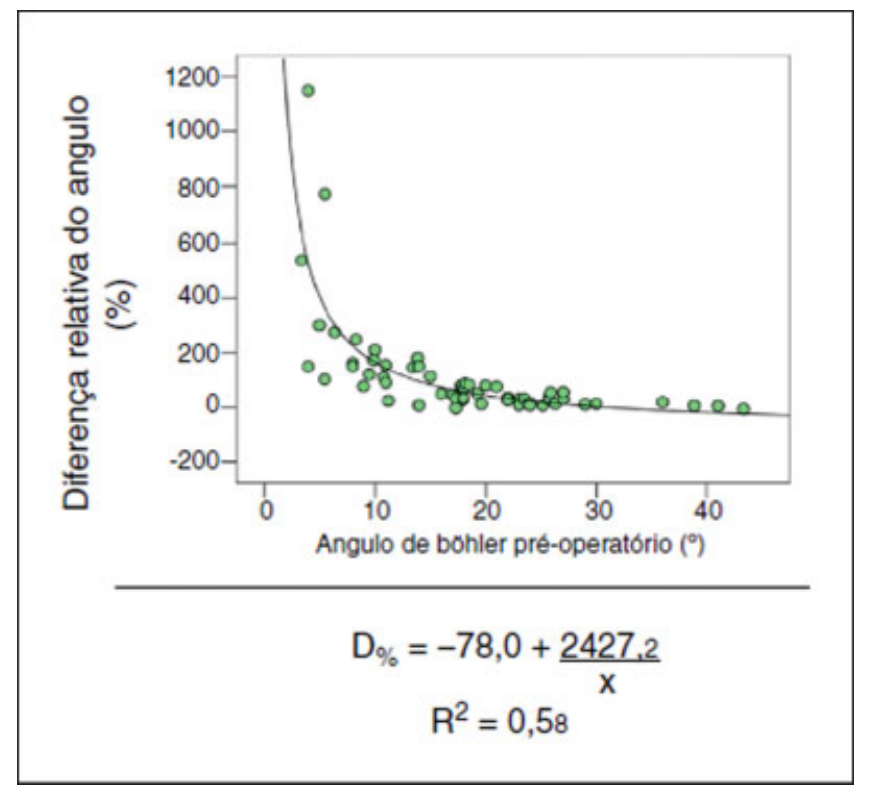

Fig. 5 Relação entre a diferença relativa do ângulo e a medida do ângulo de Böhler pré-operatório, avaliação global.

diferença relativa do $\mathrm{AB}$ assume valores de $6,0 \%$ a $80,0 \%$, com baixa variabilidade em torno da diferença média de $28,0 \%$. Quando os ângulos pré-operatórios estão fora da faixa de normalidade, a diferença relativa do $A B$ assume valores de $-6,0 \%$ a $1.150,0 \%$, com alta variabilidade em torno da diferença média de $163,2 \%$. Ou seja, se o $A B$ pré-operatório estiver na faixa de normalidade, o ângulo pós-operatório será, em média, 1,28 vez o ângulo pré-operatório; se o $A B$ pré-operatório estiver fora da faixa de normalidade, o $A B$ pós-operatório será, em média, 17,3 vezes o ângulo pré-operatório.

\section{Análise de concordância entre os avaliadores}

A - Tabela 6 demonstra a análise de concordância entre as medidas dos ângulos dos dois avaliadores. Em relação à concordância absoluta, somente em uma avaliação os dois avaliadores atribuíram a mesma medida ao ângulo. Mas a diferença entre as medidas dos dois avaliadores não ultrapassou 4 graus na medida pré-operatória e 6 graus na medida pós-operatória. No global, a concordância entre os avaliadores é excelente, tanto em relação ao valor pontual estimado, igual a 0,98 , quanto em relação ao intervalo de confiança do ICC, todo contido no intervalo de excelente concordância que vai de 0,8 a 1,0. Nas medidas pré e pós-operatórias, as estimativas de ICC pontual e intervalar mostram excelente concordância entre os dois avaliadores. Os p-valores dos testes F para os valores do ICC não são reportados aqui, mas foram todos menores do que 0,0001 , indicaram que os valores dos ICCs são todos significativamente não nulos. 
162 Ângulo de Böhler Labronici et al.

Tabela 7 Análise de concordância entre os ângulos medidos pelos dois avaliadores, para as avaliações pré e pós-operatórias e global

\begin{tabular}{|l|l|l|l|l|l|l|}
\hline \multirow{2}{*}{ Fonte da medida } & \multicolumn{3}{|c|}{ Böhler-Avaliador 1 vs Avaliador 2 } & Variação das medidas \\
\cline { 2 - 7 } & CCI & IC para o CCI & $\begin{array}{l}\text { Classificação da } \\
\text { concordância }\end{array}$ & $\begin{array}{l}\text { Concordância do teste } \\
\text { bruta }\end{array}$ & $\begin{array}{l}\text { de Student } \\
\text { pareado }\end{array}$ \\
\hline $\begin{array}{l}\text { Pré-operatória } \\
\text { (31 avaliações) }\end{array}$ & 0,98 & $0,95-0,99$ & Excelente & $1(3,2 \%)$ & $0,0-4,0$ & 0,457 \\
\hline $\begin{array}{l}\text { Pós-operatória } \\
\text { (31 avaliações) }\end{array}$ & 0,96 & $0,92-0,98$ & Excelente & $0(0,0)$ & $0,8-6,0$ & 0,595 \\
\hline $\begin{array}{l}\text { Ambas as medidas } \\
\text { (62 avaliações) }\end{array}$ & 0,98 & $0,96-0,99$ & Excelente & $1(1,6 \%)$ & $-6,0-4,0$ & 0,370 \\
\hline
\end{tabular}

Abbreviations: IC, interval de confiança; $\mathrm{CCl}$, coeficiente de correlação intraclasse.

Corroborando os resultados da análise de concordância excelente entre os dois valores, os p-valores do teste $t$ de Student, na comparação das medidas dos dois avaliadores, todas maiores do que 5,0\%, mostram que não havia diferença significativa entre as medidas dos ângulos dos dois avaliadores. A análise de concordância entre os dois avaliadores feita nesta seção mostra que ambos os avaliadores, embora diferissem em suas avaliações dos ângulos, não diferiam significativamente, apresentaram o mesmo nível de expertise em avaliar os ângulos.

\section{Discussão}

Baseado nos resultados da análise estatística deste estudo em 31 pacientes com fraturas desviadas do calcâneo, verificou-se que quanto maior o $A B$ no pré-operatório, o que significaria uma fratura com menor desvio, apresentava uma variação média de $28 \%$ (6,0\% a $80 \%$ ), o ângulo no pósoperatório será 1,28 vez o ângulo do pós-operatório. No entanto, quanto menor o $\mathrm{AB}$ no pré-operatório, o que significaria uma fratura com maior desvio, apresentava uma variação média de $162,2 \%$ (de $6,0 \%$ a $1.150,0 \%$ ), o ângulo no pós-operatório será 17,3 vezes o ângulo do pós-operatório. Ficou demonstrado que o cirurgião tem uma tendência de reduzir anatomicamente fraturas mais graves do que as que apresentam desvios menores. Este estudo também confirmou uma boa correlação interobservador para o $\mathrm{AB}$.

A maioria da literatura que analisa o $A B$ realça a restauração do ângulo e o significado do prognóstico. Apesar de alguns autores sugerirem que a restauração do $A B$ pode não ter efeito sobre os resultados, grande parte das pesquisas tem mostrado que a restauração do $\mathrm{AB}$ orienta uma redução anatômica e melhora os resultados. ${ }^{10,15-24}$ Da mesma forma, existe uma grande evidência na literatura de que o $A B$ inicial muito reduzido, nas fraturas do calcâneo, é um fator de prognóstico e um preditor de mau resultado. ${ }^{10,20,25,26}$

Este estudo usou o ângulo de corte de 20。 ou menos para definir as fraturas mais graves, baseado no trabalho de Isaacs et $a l,{ }^{27}$ que demonstraram ser o $\mathrm{AB}$ mais preciso para determinar a presença ou ausência de fratura. Suas observações sugerem que a precisão do $\mathrm{AB}$ pode torná-lo adequado como ferramenta de triagem no diagnóstico da fratura do calcâneo. Também demonstraram outro aspecto importante, que o ângulo de corte de 20。 ou menos foi independente do ângulo antes da fratura. Consequentemente, não seria necessário medir o $A B$ no lado contralateral nas fraturas do calcâneo.

Apesar de a literatura ter demonstrado que o $\mathrm{AB}$ apresenta uma boa credibilidade, muitas classificações e medidas têm se mostrado pouco confiáveis. ${ }^{28-31}$ As justificativas mais comuns incluem falta de treinamento do observador, imagens radiográficas não uniformes e de baixa qualidade e descrições vagas e imprecisas das classificações. Outra origem de dificuldade para a medição do ângulo pode ser uma sobreposição do material de síntese sobre os pontos de referências no pósoperatório. Otero et $\mathrm{al}^{32}$ demonstraram que mesmo com observadores treinados e uma adequada configuração na medida radiográfica do $A B$, divergências de interpretação são comuns. Gonzalez et $\mathrm{al}^{33}$ encontraram medição de erro de 6 graus para o $\mathrm{AB}$. Dois fatores que aumentaram o erro incluíram nível baixo de treinamento dos observadores, como aumento da obliquidade nas radiografias em incidência lateral. Observaram que essa diferença foi somente vista quando a radiografia era feita em ângulo muito oblíquo (anterior em 20。 e caudal em 15o). Para evitar discrepâncias e minimizar potenciais riscos de falha nas medidas do $A B$, as medições foram previamente definidas, usaram-se dois observadores treinados, um protocolo de medidas, o desempenho dos avaliadores foi testado e radiografias de baixa qualidade, para fazer as medições, foram excluídas.

Como descrito por Bland e Altman, ${ }^{34}$ medidas repetidas sobre o mesmo assunto variam em torno de um valor verdadeiro, por causa do erro de medição e desvio-padrão de medidas repetidas permite medir o tamanho do erro de medição. Em nosso estudo, usamos o coeficiente de variação, que significa a medida empregada para estimar a precisão de experimentos, e verificamos que as medidas do AB apresentaram alta variabilidade entre os 31 pacientes avaliados. Os resultados demonstraram, tanto para cada avaliador quanto no global da amostra, que o ângulo aumentou significativamente após a cirurgia. O ângulo do pós-operatório foi, em média 10,6 graus maior do que no pré-operatório e na variação dos ângulos entre os observadores não houve diferença significativa $(\mathrm{p}=0,761)$. Um fator interessante observado é que foi possível verificar que a diferença relativa observada no $\mathrm{AB}$ no pós-operatório foi maior para menores valores do ângulo pré-operatório e decresce à medida que o 
ângulo pré-operatório aumenta. Quanto mais distante da faixa de normalidade, de 20 a 40 graus, o ângulo préoperatório, maior a diferença relativa observada no ângulo após a cirurgia. Quando o ângulo pré-operatório está na faixa de normalidade de 20 a 40 graus, a diferença relativa do $A B$ assume valores de $6,0 \%$ a $80,0 \%$, com baixa variabilidade em torno da diferença média de $28,0 \%$. Quando os ângulos préoperatórios estão fora da faixa de normalidade, a diferença relativa do $A B$ assume valores de $-6,0 \%$ a $1.150,0 \%$, com alta variabilidade em torno da diferença média de 163,2\%. Ou seja, se o AB pré-operatório estiver na faixa de normalidade, o ângulo pós-operatório será, em média, 1,28 vez o ângulo pré-operatório; se o $A B$ pré-operatório estiver fora da faixa de normalidade, o $A B$ pós-operatório será, em média, 17,3 vezes o ângulo pré-operatório.

O coeficiente de correlação intraclasse (ICC) é uma estimativa da fracção da variabilidade total de medidas devido a variações entre os indivíduos. Otero et $\mathrm{al}^{32}$ não observaram diferença considerável no ICC para a medição do AB inter ou intraobservador tanto nas radiografias no pré-operatório como no pós-operatório. Este estudo demonstrou que analisando a concordância entre as medidas dos ângulos dos dois avaliadores a diferença entre as medidas não ultrapassou 4 graus na medida pré-operatória e 6 graus na medida pósoperatória. No global, a concordância entre os avaliadores foi excelente, (igual a 0,98). Nas medidas pré e pós-operatórias, as estimativas de ICC pontual e intervalar mostram excelente concordância entre os dois avaliadores.

Existem algumas limitações neste estudo que podem influenciar os resultados. Foi um estudo retrospectivo com número pequeno de avaliadores, as radiografias usadas foram feitas em pacientes no atendimento clínico diário, e não com objetivo de pesquisa, e isso poderia alterar e afetar as medidas do ângulo. Por isso, excluímos algumas radiografias que poderiam gerar dúvidas quanto à medição. A pesquisa não foi feita intraobservador.

\section{Conclusão}

No presente trabalho, o $\mathrm{AB}$ foi avaliado em dois momentos, pré e pós-operatório, por dois avaliadores. Na análise global, verificou-se que as medidas do $A B$ no pós-operatório são, em média, significativamente maiores do que o ângulo préoperatório. Verificou-se que a diferença relativa observada no ângulo após a cirurgia é maior para menores valores do ângulo no pré-operatório e decresce à medida que o ângulo pré-operatório aumenta. Quanto mais distante da faixa de normalidade, de 20 graus a 40 graus, estiver o ângulo préoperatório, maior a diferença no ângulo após a cirurgia. Quando o ângulo pré-operatório está na faixa de normalidade de 20 graus a 40 graus, o ângulo pós-operatório será, em média, 1,28 vez o ângulo pré-operatório; se o ângulo de Böhler pré-operatório estiver fora da faixa de normalidade, o ângulo pós-operatório será, em média, 17,3 vezes o ângulo pré-operatório. Ficou demonstrado que as fraturas mais graves apresentam melhores resultados anatômicos quando comparadas com as fraturas moderadas. Este estudo também confirmou uma boa correlação interobservador para o $\mathrm{AB}$.

Conflitos de interesse

Os autores declaram não haver conflitos de interesse.

\section{Referências}

1 Tomesen T, Biert J, Frölke JPM. Treatment of displaced intraarticular calcaneal fractures with closed reduction and percutaneous screw fixation. J Bone Joint Surg Am 2011;93(10):920-928

2 Eastwood DM, Langkamer VG, Atkins RM. Intra-articular fractures of the calcaneum. Part II: Open reduction and internal fixation by the extended lateral transcalcaneal approach. J Bone Joint Surg Br 1993;75(02):189-195

3 Johnson EE, Gebhardt JS. Surgical management of calcaneal fractures using bilateral incisions and minimal internal fixation. Clin Orthop Relat Res 1993;(290):117-124

4 Leung KS, Chan WS, Shen WY, Pak PP, So WS, Leung PC. Operative treatment of intraarticular fractures of the os calcis-the role of rigid internal fixation and primary bone grafting: preliminary results. J Orthop Trauma 1989;3(03):232-240

5 O'Farrell DA, O'Byrne JM, McCabe JP, Stephens MM. Fractures of the os calcis: improved results with internal fixation. Injury 1993; 24(04):263-265

6 Parkes JC 2nd. The nonreductive treatment for fractures of the Os calcis. Orthop Clin North Am 1973;4(01):193-195

7 Böhler L. Diagnosis, pathology, and treatment of fractures of the os calcis. J Bone Joint Surg Am 1931;13(01):75-89

8 Buckley R, Tough S, McCormack R, Pate G, Leighton R, Petrie D, et al. Operative compared with nonoperative treatment of displaced intra-articular calcaneal fractures: a prospective, randomized, controlled multicenter trial. J Bone Joint Surg Am 2002;84-A(10): 1733-1744

9 Dooley P, Buckley R, Tough S, McCormack B, Pate G, Leighton R, et al. Bilateral calcaneal fractures: operative versus nonoperative treatment. Foot Ankle Int/Am Orthop Foot Ankle Soc/Swiss Foot Ankle Soc 2004;25(02):47-52

10 Loucks C, Buckley R. Bohler's angle: correlation with outcome in displaced intra-articular calcaneal fractures. J Orthop Trauma 1999;13(08):554-558

11 Basile A. Operative versus nonoperative treatment of displaced intra-articular calcaneal fractures in elderly patients. J Foot Ankle Surg 2010;49(01):25-32

12 Maskill JD, Bohay DR, Anderson JG. Calcaneus fractures: a review article. Foot Ankle Clin 2005;10(03):463-489, vi

13 Hyer CF, Atway S, Berlet GC, Lee TH. Early weight bearing of calcaneal fractures fixated with locked plates: a radiographic review. Foot Ankle Spec 2010;3(06):320-323

14 Knight JR, Gross EA, Bradley GH, Bay C, LoVecchio F. Boehler's angle and the critical angle of Gissane are of limited use in diagnosing calcaneus fractures in the ED. Am J Emerg Med 2006;24(04):423-427

15 Di Schino M, Bensaïda M, Vandenbussche E, Augereau B, Nich C. [Results of open reduction and cortico-cancellous autograft of intra-articular calcaneal fractures according to Palmer]. Rev Chir Orthop Repar Appar Mot 2008;94(02):135-144

16 Cave EF. Fracture of the os calcis-the problem in general. Clin Orthop Relat Res 1963;30(30):64-66

17 Hildebrand KA, Buckley RE, Mohtadi NG, Faris P. Functional outcome measures after displaced intra-articular calcaneal fractures. J Bone Joint Surg Br 1996;78(01):119-123

18 Kitaoka HB, Schaap EJ, Chao EY, An KN. Displaced intra-articular fractures of the calcaneus treated non-operatively. Clinical results 
164 Ângulo de Böhler Labronici et al.

and analysis of motion and ground-reaction and temporal forces. J Bone Joint Surg Am 1994;76(10):1531-1540

19 Lindsay WR, Dewar FP. Fractures of the os calcis. Am J Surg 1958; 95(04):555-576

20 Paley D, Hall H. Intra-articular fractures of the calcaneus. A critical analysis of results and prognostic factors. J Bone Joint Surg Am 1993;75(03):342-354

21 Pillai A, Basappa P, Ehrendorfer S. Modified Essex-Lopresti / Westheus reduction for displaced intra-articular fractures of the calcaneus. Description of surgical technique and early outcomes. Acta Orthop Belg 2007;73(01):83-87

22 Hutchinson F III, Huebner MK. Treatment of os calcis fractures by open reduction and internal fixation. Foot Ankle Int 1994;15(05): 225-232

23 O'Brien J, Buckley R, McCormack R, Pate G, Leighton R, Petrie D, et al. Personal gait satisfaction after displaced intraarticular calcaneal fractures: a 2-8 year followup. Foot Ankle Int 2004;25(09): 657-665

24 Jiang SD, Jiang LS, Dai LY. Surgical treatment of calcaneal fractures with use of beta-tricalcium phosphate ceramic grafting. Foot Ankle Int 2008;29(10):1015-1019

25 Shuler FD, Conti SF, Gruen GS, Abidi NA. Wound-healing risk factors after open reduction and internal fixation of calcaneal fractures: does correction of Bohler's angle alter outcomes? Orthop Clin North Am 2001;32(01):187-192, x

26 Csizy M, Buckley R, Tough S, Leighton R, Smith J, McCormack R, et al. Displaced intra-articular calcaneal fractures: variables predicting late subtalar fusion. J Orthop Trauma 2003;17(02):106-112
27 Isaacs JD, Baba M, Huang P, Symes M, Guzman M, Nandapalan H, et al. The diagnostic accuracy of Böhler's angle in fractures of the calcaneus. J Emerg Med 2013;45(06):879-884

28 Neyt JG, Weinstein SL, Spratt KF, Dolan L, Morcuende J, Dietz FR, et al. Stulberg classification system for evaluation of Legg-CalvéPerthes disease: intra-rater and inter-rater reliability. J Bone Joint Surg Am 1999;81(09):1209-1216

29 Andersen DJ, Blair WF, Steyers CM Jr, Adams BD, el-Khouri GY, Brandser EA. Classification of distal radius fractures: an analysis of interobserver reliability and intraobserver reproducibility. J Hand Surg Am 1996;21(04):574-582

30 Carman DL, Browne RH, Birch JG. Measurement of scoliosis and kyphosis radiographs. Intraobserver and interobserver variation. J Bone Joint Surg Am 1990;72(03):328-333

31 Bernstein J, Adler LM, Blank JE, Dalsey RM, Williams GR, Iannotti JP. Evaluation of the Neer system of classification of proximal humeral fractures with computerized tomographic scans and plain radiographs. J Bone Joint Surg Am 1996;78(09):1371-1375

32 Otero JE, Westerlind BO, Tantavisut S, Karam MD, Phisitkul P, Akoh CC, et al. There is poor reliability of Böhler's angle and the crucial angle of Gissane in assessing displaced intra-articular calcaneal fractures. Foot Ankle Surg 2015;21(04):277-281

33 Gonzalez TA, Ehrlichman LK, Macaulay AA, Gitajn IL, Toussaint RJ, Zurakowski D, et al. Determining measurement error for Bohler's angle and the effect of X-ray obliquity on accuracy. Foot Ankle Spec 2016;9(05):409-416

34 Bland JM, Altman DG. Measurement error. BMJ 1996;313 (7059):744 